\title{
Interactive Maths with GeoGebra
}

\author{
$\underline{\text { doi:10.3991/ijet.v6iS1.1620 }}$ \\ Daniela Velichová \\ Slovak University of Technology, Bratislava, Slovakia
}

\begin{abstract}
This paper brings information on dynamic multiplatform mathematical software GeoGebra produced by Markus Hohenwarter in 2002 for learning and teaching mathematics, available free on web in 45 languages. GeoGebra enables production of self-standing dynamic worksheets as interactive java applets embedded in html pages presenting dynamic constructions and interactive calculations on Internet. Thus it is enhancing available powerful electronic tools suitable for production of instructional materials in on-line maths education. Several possibilities are presented on how this useful utility might be used in e-learning solutions as a dynamic interactive platform for calculations and drawings.
\end{abstract}

Index Terms-dynamic mathematical software, cognitive tools, cognitive connections, symbolization in mathematics.

\section{INTRODUCTION}

Research in cognitive psychology indicates that our brains store knowledge using both words and images. Instruction that targets and engages both of these systems of representation has been shown to significantly increase students' comprehension and retention. Explicitly engaging students in the creation and usage of non-linguistic representations has even been shown to stimulate and increase activity in the brain. Manipulatives are concrete or symbolic artefacts that students interact with while learning new topics. They are powerful instructional aids because they enable active, hands-on exploration of abstract concepts. Research has shown that computer-based manipulatives are even more effective than ones involving physical objects, in part because they can dynamically link multiple representations together.

At the beginning of the $3 \mathrm{rd}$ millennium we are facing a dramatic change in the basic nature of teaching and learning strategies caused by the massive use of new technology. We can benefit from this development in general, and in mathematics especially, as currently available dynamic and visual learning environments could affect our perspective in terms of the content and comprehension of mathematics education as well. There is an increasing importance of dynamic linking of multiple representations in facilitating students' visualization because students can explore, solve, and communicate mathematical concepts in various ways, such as using dynamic multiple representations and mathematical modelling. Simply showing pictures or figures is not sufficient to encourage students to visualize or use various representations. Extensive usage of new communication technologies has enlarged the pool of cognitive tools and possibilities of their application in teaching and learning processes with visible consequences and feasible improvements.

Cognitive tools have their irreplaceable position in the didactics and educational theory and there exist a lot of ideas on how we can understand their role in education.
Cognitive tools allow users to explore mathematical concepts dynamically and have been increasingly discussed in the recent years, as can be seen in [1] - [5]. Authors Luis Moreno-Armella, Stephen J. Hegedus, and James J. Kaput provide in [4], p. 103 an historical overview of symbolization, which can be considered as a kind of the evolution of cognitive tools. They identify five stages of the evolution of symbolization "from static, inert inscriptions to dynamic objects or diagrams that are constructible, able to be manipulated and interactive".

The "static inert stage" of symbolization in mathematics consists of a wide spectrum of textbooks and handouts prepared by teachers for ages and still widely used. The second stage denoted as "static kinaesthetic/aesthetic stage", dwells in the use of chalk and marker pens on black-green-white boards and slides with overhead projectors and allows users to erase and change the inscription. Besides the flexibility to make changes, these tools also provide opportunities to use several colours to emphasize particular notations. In the third "static computational stage" users get a static presentation of the input they provide to some certain devices such as calculators and graphing tools. Gabriel Salomon [6] favours this type of interaction with cognitive tools because he believes that users can have more opportunities to deal with high order thinking tasks if they leave low level cognitive activities to the tools. The last two stages of this evolution framework focus on the dynamic perspectives of maths presentation. In the fourth, "discrete dynamic stage" a discrete co-action between user and environment is described. The users have more control over the process and the output obtained from the media, because they have the ability to change and to manipulate the input dynamically through the first interactive tools that have recently appear in some mathematics software solutions. In the fifth stage named "continuous dynamic stage" users can be actively interacting with the real objects through dynamic interactive devices and therefore they can get instantaneous and continuous feedback.

The dynamic worksheets prepared in GeoGebra enable users to create dynamic mathematical objects such as graphs and functions and to interact with these objects. Assuming that these mathematical objects are the real objects of this platform, although the platform itself is virtual in nature, then the continuous interaction between these objects and users could be considered as a continuous dynamic interaction. Similarly, GeoGebra can be considered as a haptic device detecting the movements of the slider (that is controlled by the tactile connection of the user and the computer mouse) and adjustable objects described in this platform, as mentioned by Karadag and McDougall in [7]. 


\section{SPECIAL FOCUS PAPER \\ INTERACTIVE MATHS WITH GEOGEBRA}

\section{GeoGebra Features AND UsAGE}

GeoGebra is an intuitively controllable application suitable for all users without any extra mastership in computer literacy. It can serve for development of instructional materials in mathematics in many different forms, types and styles, and for all levels of mathematical education. It is free to be downloaded form a website [10], while nothing but a Java 6 platform is necessary for its full operation. GeoGebra seems to be particularly easy and intuitive to learn. Files can be saved in ".ggb" format, or as dynamic web pages. GeoGebra can output files as pictures (.png) or as encapsulated postscript for publication quality illustrations. GeoGebra user interface offers a rich graphics menu for drawing various objects, while the complete construction protocol is saved and it appears in any chosen language from the available 45 versions, therefore no translation is necessary and free sharing of developed instructional materials is genuinely supported all over the world. Users are encouraged to visit GeoGebra webpage and GeoGebra user's forum GeoGebraWiki, a free pool of teaching materials for this dynamic mathematics software where everyone can contribute and upload materials.

GeoGebra has a built-in Cartesian coordinate system, and accepts both geometric commands (drawing a line through two given points, a conic section determined in different ways, in Fig 1. by 5 points) and algebraic ones (drawing a curve with a given equation). Among its more interesting features is the ability to draw tangent lines to algebraic and even transcendental curves at given points, while equation of this tangent line is available immediately too. This double representation: the geometric synthetic one and the algebraic-analytic one is one of the greatest advantages of GeoGebra software that mostly suits to didactic aims of full comprehension of basic mathematical concepts. One can enter the objects either as geometric objects (via drop down menus) or as algebraic objects - pairs of coordinates, functions - via the entry line. Moving the objects in the Geometry window (see Fig. 1.) changes the expressions in the Algebra window accordingly (on the left in Fig. 1.), and vice-versa, editing the expressions in the Algebra Window results in the respective change in the Geometry Window. This is the main feature of GeoGebra meeting the demands of many didactics and educators to provide for the students as many representation forms as possible.

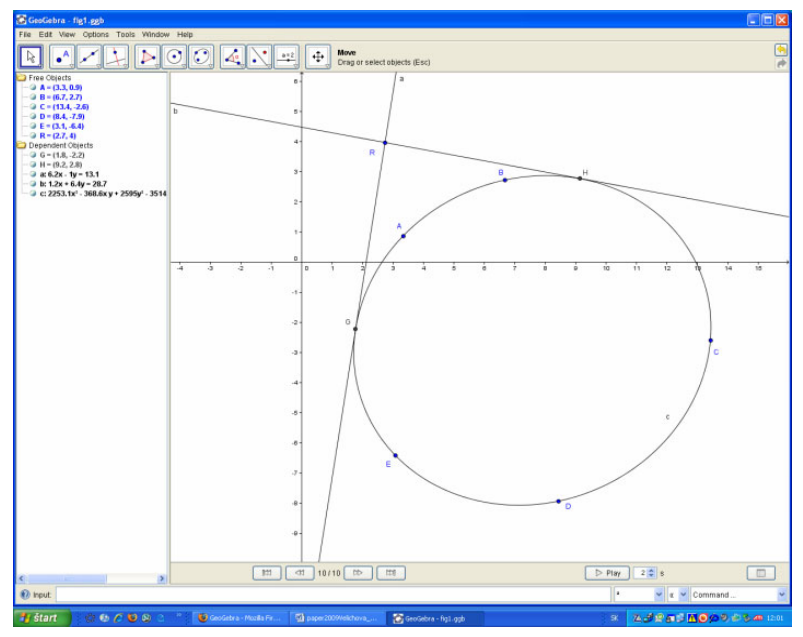

Figure 1. GeoGebra user interface
Teachers can guide students to understand how these two representations of mathematical objects are connected to each other. Changes in the superposition of the conic section determining points are reflected in the form of its equation. Following these changes in the algebraic window students can realise the role of the coefficients of the bi-quadratic form. Existence of the tangent line to the conic section passing through the chosen point can be investigated by changing its position, while equations of the tangent lines appear immediately in the updated form.

In this way, students are engaged to study relations of mathematical objects in more complex form, in both ways, geometric - synthetic and algebraic - analytic representations receiving thus comprehensive knowledge.

After having developed an understanding of the relation between the particular type of determined conic section and its equation, and tangent line to a conic section and its equation, students have the opportunities to create cognitive connections between their previous knowledge and the outcomes of the current exploration. They can create various cognitive connections, and by further explorations they can be engaged in asking what-if questions, as: What if would two of the conic section determining points coincide? What do I have to change in the 5 determining point's superposition in order to receive ellipse, parabola, or hyperbola? What if I move point $\mathrm{R}$ to be the point on the conic section or to be its interior point?

Many various questions that can be created by teachers and students are leading to heuristic learning approaches, hands-on exploration of abstract concepts and cognitive connections. GeoGebra represents in this sense the example of a cognitive tool of the fifth, continuous dynamic stage, as users are really interacting with the real objects through dynamic interactive devices and they receive instantaneous and continuous feedback in two simultaneous forms.

Teachers can prepare suitable applications for demonstration of particular mathematical concepts, and students can work directly with these supported materials. But they are also encouraged to create their own applications, developing thus deeper insight and comprehensive cognitive connections, and cultivating their creativity and imagination. They can also contribute this way to the development of new variegated instructional materials and worksheets for the overall usage and share in the classroom. Consequences of this approach are extremely important and significant, as the usage of dynamic cognitive tools influences the former passive role of students in the process of education. Now they become actively involved in the process, acting not only as self-learners, but also in the role of demonstrators for other students in the classroom.

\section{GeOMETRIC Functions AND TOOLS As FunCtions}

GeoGebra separates mathematical objects into free objects and dependent objects. Where the dependent objects are defined by an explicit construction (algebraic or geometric) the construction steps can be encapsulated into a tool. Once the tool has been defined a new button appears on the tool bar and a corresponding function name is available to the user. Such tools are essentially functions, and may operate with geometric objects such as circles, lines and points. The tools function within GeoGebra is 


\section{SPECIAL FOCUS PAPER \\ INTERACTIVE MATHS WITH GEOGEBRA}

interesting because it allows geometrical functions to be defined, which illustrate a key mathematical process: encapsulation or compression. Using these tools it is possible to extend the software in natural ways, just as mathematical domains are extended during normal teaching.

The idea of a tool as a function appears in paper by Sangwin, [9]. It allows the user to behave mathematically in two important ways: compression and extension. Compression or encapsulation is the process by which a mathematician takes a complex procedure or construction and represents it by a single step. Simple example of compression is arithmetic multiplication: repeated addition is compressed to a single step. Geometrical example of compression is a theorem of Euclidean Geometry that given three points which are not-collinear a unique circle can be determined through them. This multi-step construction, which requires drawing perpendicular bisectors to line segments, can be encapsulated into a single operation. GeoGebra of course provides a button to do sot. For mathematicians it is interesting to notice that this compression construction process results in a well defined geometrical function: take three points and return a circle (or a straight line).

Users are allowed to create their own tools as macroconstructions, while buttons for calling these geometric functions are immediately available in the basic GeoGebra menu. This is one of the features that are extremely convenient in the view of customers, who are free in customisation of the software interface menus according to their needs.

Dynamic constructions are one of the most powerful GeoGebra features. Users are able to create dynamic worksheets demonstrating certain properties and features generally applicable to specific basic geometric objects and dynamically change the form of these objects. Properties of tangent lines to parabola and position of points symmetric to the parabola focus with respect to all parabola tangent lines are illustrated in Fig. 2.

Construction is stored in a construction protocol, available on request form the view menu, and can be followed step by step in there. Construction protocol appears in the chosen language of the GeoGebra interface that leads to no need of its translation to other languages. In addition, the whole construction can also be re-played via the navigation bar for construction steps in step-by-step mode, while the choice of automatic play is available with the offer of customisable timing set up.

Other example from mathematics can be the explanation of the concept of a function derivative and its geometric meaning. This basic concept form the mathematical analysis is very often underestimated as rather easy to understand and visualise. Anyhow, dynamic illustration (Fig. 3.) of the changing slope of the tangent line to the graph of a function of one real variable according to the value of the function first derivative in the respective point is rather crucial for the overall understanding of the function behaviour. It is easy to illustrate intervals on which function is defined and to estimate intervals, where function is increasing (positive slope) or decreasing (negative slope), and to find points, in which the first derivative equals to zero, therefore function is reaching the extremes in there, its maximum or minimum value. Consequently, tangent line in the point of extreme ap-

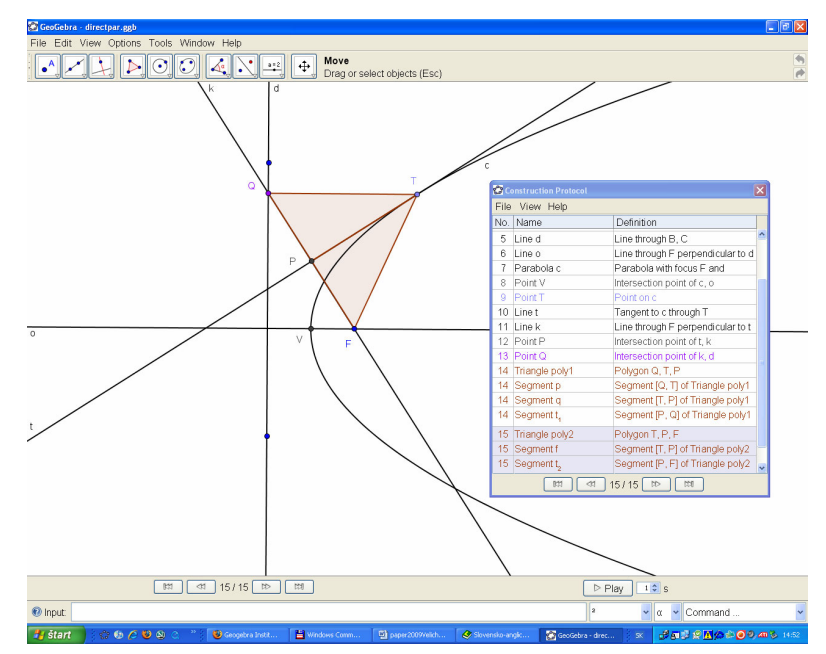

Figure 2. GeoGebra dynamic construction

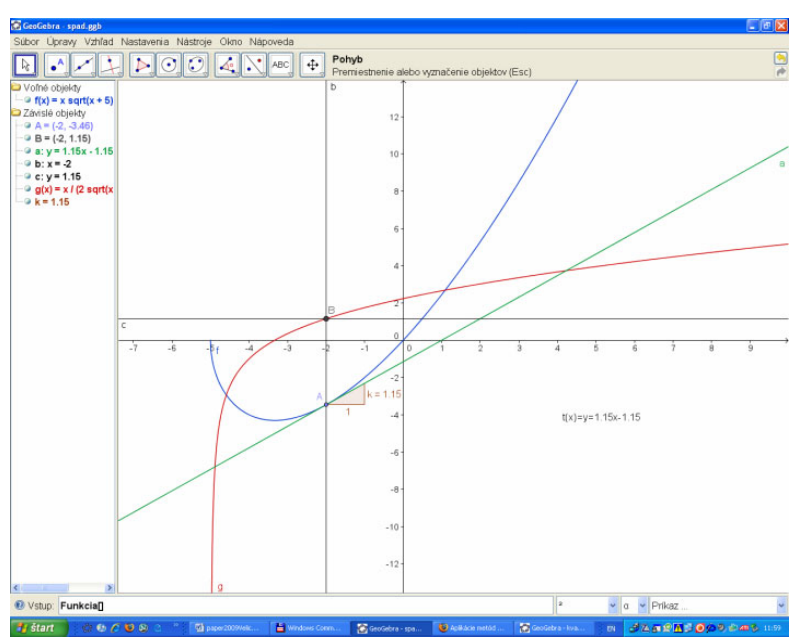

Figure 3. Geometric meaning of a function derivative

pears as parallel to the coordinate axis $x$. Also points, in which the first derivative of the function is not defined, can be illustrated also, as the slope of tangent line to the function graph in such point goes to infinity and tangent line in this point to the function graph is perpendicular to coordinate axis $x$. The concept of the global extremes and maximal and minimal value of a function of one variable on the given interval can be dynamically presented and investigated clearly.

\section{APPLICATIONS FOR ON-LiNE USAGE}

Apart from the standalone application, GeoGebra also allows the creation of interactive web pages with embedded java applets [8]. These targeted learning and demonstration environments are freely shared by mathematics educators on collaborative online platforms like the GeoGebraWiki [10]. The number of visitors to the GeoGebra website has increased since 2004 from 2000 per month to over 300,000 per month coming from over 180 countries. The International GeoGebra institute has been established, coordinating the work of thousands of volunteers all over the world in the structure of accredited national GeoGebra institutes in different countries.

GeoGebra dynamic spreadsheets enable to produce interactive presentations directly on the web serving as electronic instructional sources for e-learning solutions in the form of dynamic cognitive tools. These html pages 


\section{SPECIAL FOCUS PAPER \\ INTERACTIVE MATHS WITH GEOGEBRA}

can be used directly from Internet and are presentable in all common web-browsers, while there is no need to install GeoGebra software in the user's computer. The only necessary prerequisite in addition to the web-browser is the installed Java support that is essential.

Illustration in Fig. 4. is from the Slovak database of elearning materials at the GeoGebra Institute of Slovakia [11]. Properties of the ellipse control circle are presented here, while the geometric function locus is used here to demonstrate the form of the set of all points that are symmetric to one focus of an ellipse with respect to its tangent lines. Moving the tangent point $T$ on the ellipse, symmetric points $Q$ appear and draw the control circle. According to the specific setting chosen for the export of the source GeoGebra file ggb into the on-line presentable $\mathrm{html}$ file, button for construction protocol, navigation bar for construction steps and play button with timing set-up window can be visible in the dynamic spreadsheet application. The entire construction can be then replayed and followed step-by-step. Interactive approach on the dynamic spreadsheet can then be used for animation of the tangent point movement along the ellipse, while determined control circle is generated as locus of symmetric points Q.

A collection of similar dynamic instructional materials is available also in the European Virtual Laboratory of Mathematics, in English from the EVLM Central Portal [12], in the Central EVLM database at the address www.evlm.stuba.sk/databasemenu/menu files/frame.htm, and in Slovak language from the Slovak EVLM portal [13], in the Slovak EVLM database at the address http://slovak.evlm.stuba.sk/elearning/.

Many of applets presented in these databases enable users to act interactively and to directly manipulate received results using sliders. Scope and position of the sliders are determined by applet authors but might be kept free; therefore user can move them freely in the drawing worksheet. These enable smooth change of shaping parameters and can be set to automatic change, creating thus a real animation available on web. Manipulated by users themselves, these sliders serve for exploring relations between different parameters of any object and finding their role in the determination of the shape of its representation and visualisation as geometric figure, analytic expression, equation, mathematical formula, or any logic relation and statistical table.

In Fig. 5 and 6, illustrations of dynamic applets are given, which serve for investigation of a generation of trochoidal curves as trajectories of a point moving in two simultaneous revolutions about given centres. This movement can be simply interpreted as a movement of a circle rolling without slipping around a fixed circle. An epitrochoidal curve in Fig. 5 is a roulette traced by a point attached to the rolling circle. Three available sliders enable users to change parameters for the number of epitrochoidal curve knots and cusps in the case of epicycloidal curve, distance of the moving point from one centre of revolution (centre of the moving circle) and distance of the two centres of revolutions (centres of moving and fixed circles). Applet may contain few explanatory words that introduce the illustrated object, while user is free to manipulate sliders and investigate their role in determining the modelled object shape. Hypocycloidal curves might be studied in applet illustrated in Fig. 6, where a

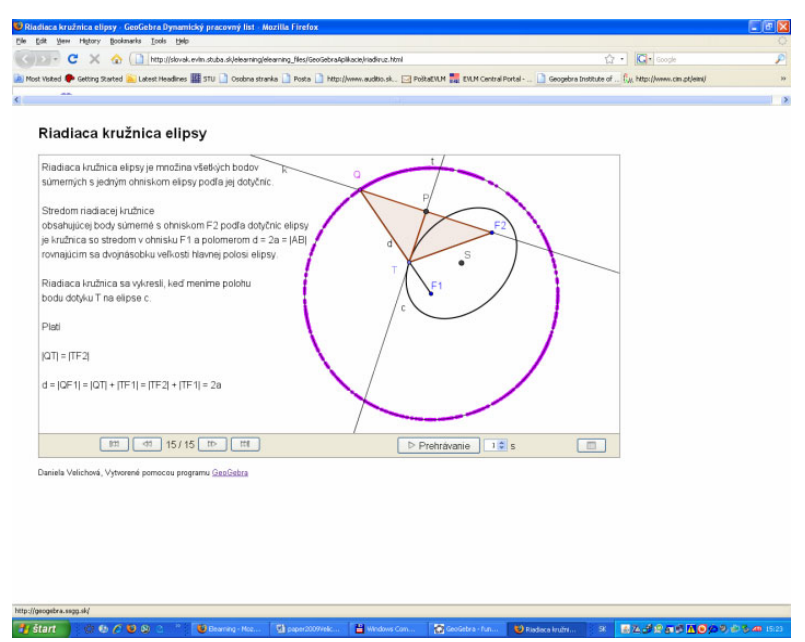

Figure 4. GeoGebra dynamic web application

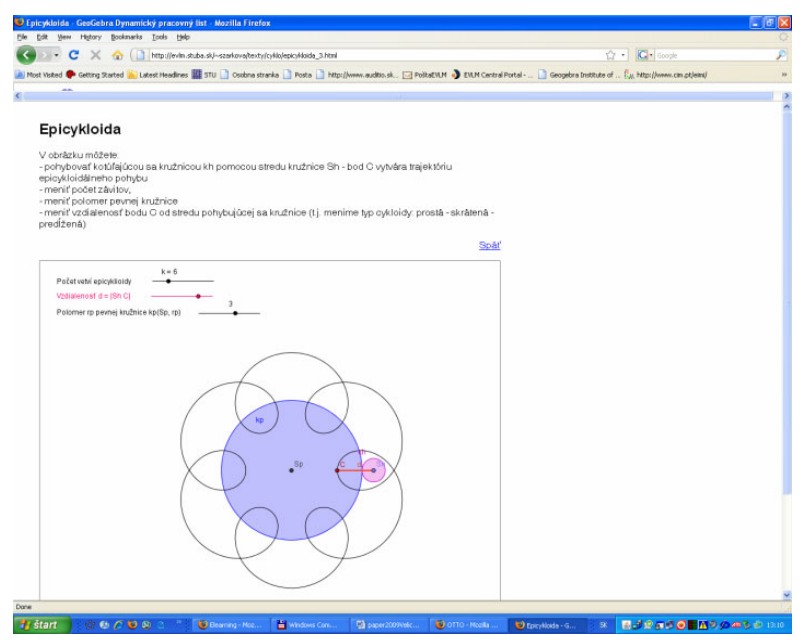

Figure 5. GeoGebra dynamic modelling of epitrochoids

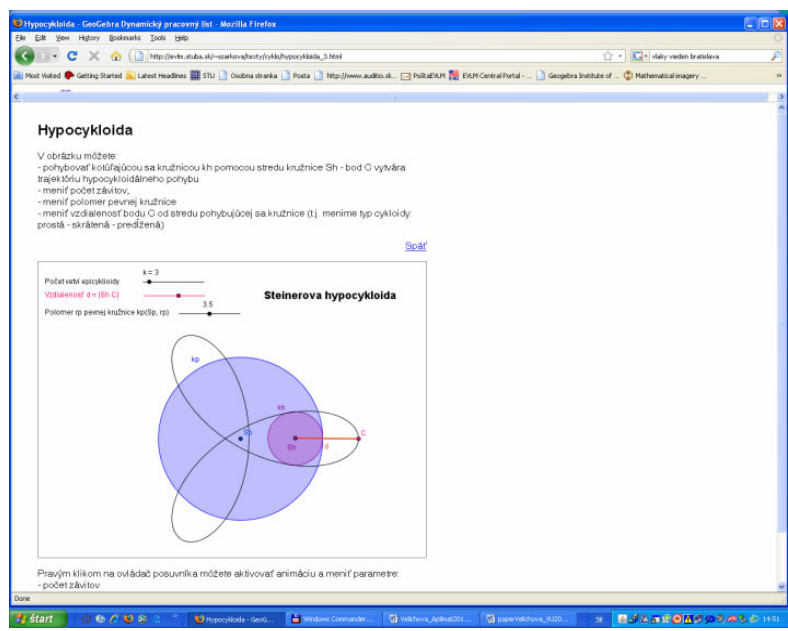

Figure 6. GeoGebra dynamic modelling of hypocycloids

Steiner hypocycloid is created as trajectory of a point attached to the circle rolling inside a fixed circle.

Collection of dynamic apples created in GeoGebra that are included in the module "Cycloidal curves" available form the Slovak EVLM database at the Slovak EVLM Portal [13] is extensively utilized by students at the Faculty of Mechanical Engineering, Slovak University of Technology in Bratislava, in both regular and distance 
learning classes. Module consists of theoretical part introducing different kind of trochoidal curves, their synthetic determination and geometric properties, and their equations. Curves are visualized in GeoGebra applets, where students can be freely exploring influence of separate parameters to the final form of the dynamically manipulated trochoidal curve. Students form distance learning classes particularly appreciate this interactive approach and availability of all materials free on-line, where they are accessible directly without any special requirement to the user computer.

Therefore the available possibility in GeoGebra to export files as applets in the form of html files is an extremely important feature and a strong advantage. This feature puts GeoGebra to a much favourite position among other software solutions, where user must have more deep knowledge of the software to receive the same effect. Some exceptions might be considered with respect to webMathematica java applets - jsp files and Mapplets developed in Maple. Anyhow, these applications do not provide so many manipulative features as GeoGebra solution, as for instance development of a dynamic drawing workplace, which is uniquely possible in GeoGebra only, without any problems. Prospective authors using other software solutions for development of dynamic materials must in addition bye the respective commercial software products that are relatively quite expensive. Certain technical problems and compatibilities still remain unsolved.

In the current GeoGebra version, Latex coding of mathematical formulas is used for representation of specific mathematical symbols, while export of the file to Latex file is possible. One of the features that GeoGebra users would like to find in this free-ware is the possibility to export GeoGebra ggb files into xml files with embedded MathML coding of mathematical formulas. This would bring a complete versatility into presentation of materials with mathematical contents on the web, as MathML coding brings semantics to maths formulas, whereas these can be easily copied and transported to widely use mathematical software applications and to be processed there.

\section{CONCLUSIONS}

Concerning the software development of GeoGebra, authors are always looking for talented Java programmers with good ideas for new features and extensions. With the recent addition of a spreadsheet view, GeoGebra is ready for more statistics charts, commands, and new tools. The upcoming computer algebra view will provide even more applications of the software at the university level. With all these planned new features, it will be crucial to focus on keeping the software's user interface simple and easy.

Thus authors are also working on a highly customizable new interface where users can easily change perspectives (e. g. from "geometry" to "statistics") and rearrange different parts of the screen using drag and drop. Team of people is also working on introducing $3 \mathrm{D}$ geometry into the GeoGebra, in the similar dynamic mode as it currently works for the case of $2 \mathrm{D}$. In the future we can expect to have a universal tool for math education combining Dynamic Geometry, Computer Algebra, semantics of mathematical formulas, dynamic 2D drawing worksheet and possibly 3D Dynamic Geometry that will hopefully keep its simplicity and user friendliness.
Finally, it should be mentioned that simple drawing of mathematical objects and figures is not enough for the building of a comprehensive understanding of basic mathematical concepts. On the other hand, creative dynamic activities are essential also to the development of one's technological content knowledge. This seems to be consistent with the general notion of mathematical understanding as one's growing competence to navigate through various representations of mathematics concepts illustrated dynamically. GeoGebra seems to be a didactic tool supporting these efforts to a high level, and in an easy, natural and user friendly way. These characteristics do predetermine this free-ware application to be used in teaching, learning and exploring mathematics into such a large extent as possible. Not surprisingly to the benefits and satisfaction of all groups of its users, teachers and students including.

\section{REFERENCES}

[1] C. Hoyles, R. Noss, "Next steps in implementing Kaptur's research programme", Educational Studies in Mathematics, Vol. 68, pp. 85-97. doi:10.1007/s10649-007-9102-4

[2] J. J. Kaput, "Technology and mathematics education”, Handbook of Research on Mathematics Teaching and Learning, The National Council of Teacher of Mathematics.

[3] D. W. Massaro, N. Cowan, "Information processing models: Microscopes of the mind", Annual Review of the Psychology, Vol. 44, pp. 383-425. doi:10.1146/annurev.ps.44.020193.002123

[4] A. Moreno, L. Armella, S. J. Hegedus, J. J. Kaput, "From static to dynamic mathematics: Historical and representational perspectives", Educational Studies in Mathematics, Vol. 68, pp. 99-111. doi:10.1007/s10649-008-9116-6

[5] R. D. Pea, "Practices of distributed intelligence and designs for education", Distributed cognitions: Psychological and educational considerations, Cambridge University Press.

[6] G. Salomon, "No distribution without individual's cognition: a dynamic interactional view", Distributed Cognition: Psychological and Educational Considerations, Cambridge University Press.

[7] Z. Karadag, D. McDougall, "Dynamic worksheets: visual learning with the guidance of Polya", MSOR Connections, Vol. 9, No 2, May - July 2009.

[8] M. Hohenwarter, Z. Lavicza, "The strength of the community: how GeoGebra can inspire technology integration in mathematics teaching", MSOR Connections, Vol. 9, No 2, May - July 2009.

[9] C. J. Sangwin, "Geometrical functions: tools in GeoGebra", MSOR Connections, Vol. 8, No 4, November 2008 - January 2009.

[10] GeoGebra webpage. [On-line]. Available: http://www.geogebra. org/cms/.

[11] Geogebra Institute of Slovakia homepage. [On-line]. Available: http://geogebra.ssgg.sk/.

[12] European Virtual Laboratory of Mathematics, Central Portal and central EVLM Database. [On-line]. Available: http://www. evlm.stuba.sk.

[13] Európske virtuálne laboratórium matematiky, Slovenský portál a Slovenský databázový portál. [On-line]. Available: http://slovak. evlm.stuba.sk/portal/.

\section{AUTHOR}

D. Velichová (daniela.velichova@stuba.sk) is professor of applied mathematics at the Faculty of Mechanical Engineering, Slovak University of Technology in Bratislava, Slovakia. Her research interests cover, among others, also new didactic methods in teaching mathematics to engineering students by means of ITC, interactive dynamic tools in particular.

This article was modified from a presentation and paper at The 10th International Virtual University Conference, in 10-11 December 2009, Bratislava, Slovak Republic.Received March $28^{\text {th }}$, 2011. Published as resubmitted by the author April $20^{\text {th }}, 2011$. 